\title{
Reactive Balance Control in Walking based on a Bipedal Linear Inverted Pendulum Model
}

\author{
Federico Parietti and Hartmut Geyer
}

\begin{abstract}
Dynamic balance depends on proper foot placement in legged locomotion and corresponding placement strategies have mainly been developed using the linear inverted pendulum model as theoretical framework. While this model can identify single leg strategies for balance control, it does not consider the double support that is common to bipedal locomotion, indicating that current strategies do not fully exploit the theoretical potential of balance control in bipedal systems. Here we extend the linear inverted pendulum model to a bipedal system which includes double support dynamics, and derive a reactive balance controller based on foot placement and double stance length. We show that this controller enables the model to stand and walk at user-defined target speeds, to transition between these behaviors by acceleration and deceleration, and to react to intermittent disturbances and compensate for permanent ones, as long as they are compatible with the swing leg dynamics placing the feet. Finally, we discuss how the versatility of this balance controller depends on including double support and suggest further steps to improve dynamic balance control in bipedal systems.
\end{abstract}

\section{INTRODUCTION}

Balance is critical to legged locomotion. Without balance, legged systems cannot hold their body upright and fall after a trip, slip, push or a similar dynamic disturbance. For example, falls connected to the loss of balance are a major cause of injury or death in the elderly population [1]. As a consequence, understanding dynamic balance has developed into a very active field of research in biomechanics, neuroscience and robotics [1] [2] [3] [4].

Three main balancing strategies have been identified for humans, comprising the ankle, hip and foot placement strategies. The first strategy uses ankle torques to counteract a lateral push in stance [2] [5] [6]. As long as the center of pressure remains within the base of support, the area between the feet on the ground, this strategy suffices. Otherwise, humans add the hip strategy, which combines fast rotations of the torso and arms to create a net torque around the center of mass (COM) that temporarily increases the horizontal force resisting a lateral push [6] [7] [8] [9]. For large disturbances, however, the two strategies do not compensate enough and the only option left to maintain balance is to take one or more corrective steps, entering locomotion [9] [10]. In locomotion, the ankle strategy looses importance [1] and the hip strategy is confined to scenarios with constrained foot placements like rope walking, as the torso is preferably kept upright [3] [9];

F. Parietti is supported by a graduate scholarship of the Alta Scuola Politecnica. $\mathrm{He}$ is with the Department of Mechanical Engineering, Politecnico di Milano, 20156 Milan, Italy, federico.parietti@mail.polimi.it.

$\mathrm{H}$. Geyer is with the Robotics Institute, Carnegie Mellon University, Pittsburgh, PA 15213, USA, hgeyer@cs. cmu. edu. thus, dynamic balance in human locomotion mainly relies on proper foot placement [11] [12] [13] [14].

The main conceptual model used to understand foot placement strategies in bipedal locomotion is the linear inverted pendulum model (LIPM) [15]. This model contracts the body to a point mass that travels on a single massless leg, which provides a vertical force equal to one body weight (Figure 1A). For very small excursions from the upright position, the LIPM approximates an inverted pendulum; for larger excursions the leg compresses and extends to accommodate the geometric constraint of a horizontal motion of the point mass at a constant height.

The LIPM has primarily been used to identify capture points for stopping locomotion (also referred to as extrapolated center of mass [13]). A capture point is the foot placement of the LIPM in response to the current speed $v_{0}$ which allows to dissipate during the subsequent leg compression the kinetic energy given by that speed and to stop in the upright position [3] [16]. If the model cannot stop within one step, it can be used to find capture points recursively, planning for several steps [10]. In addition, the model has been extended by a flywheel that resembles the hip strategy, which, by imposing an optimized torque profile on the flywheel, improves the model's stopping capabilities [3].

Other models that have been developed to derive foot placements for dynamic balance in locomotion share similar strategies with those developed for LIPM. For instance, to eventually stop, the foot placement estimator [17] places the feet in inverted pendulum locomotion to adjust impact losses to the required energy dissipation. For another example, in complex humanoid models [18] [19], foot placements are implemented as feedback control that depends on the current horizontal position and speed of the COM.

Common to these balance strategies based on the LIPM is that they derive from considering only the single leg stance. Double support is either neglected or considered as a kinematic constraint on successive single stances without control authority [20] [21] [22]. However, double support constitutes about 20 percent of the normal gait cycle in human walking [23], and it is the phase in which humans actually come to a stop. While the latter suggests that the unstable upright position in single stance may not be the control reference point in humans, the former indicates that currently proposed foot placement strategies do not fully exploit the theoretical potential of dynamic balance control in bipedal locomotion.

Here we extend the LIPM to a bipedal system which includes double support and derive a dynamic balance con- 
troller based on foot placement and double stance length. We show that this controller enables the model to stand and walk at user-defined target speeds, to transition between these behaviors by acceleration and deceleration, and to react to intermittent disturbances and compensate for permanent ones, as long as they are compatible with the swing-leg dynamics placing the feet. Finally, we discuss how the versatility of this balance controller depends on including double support and suggest further steps to improve dynamic balance control in bipedal systems.

\section{Bipedal Linear Inverted Pendulum Model}

Like the one-legged LIPM, our bipedal extension bLIPM represents the body as a point mass moving along the horizontal axis at height $y_{0}$. In single support (Fig. 1A), the stance leg (red solid line) produces a total force whose vertical component is $m g$ maintaining the constant height $y_{0}$. As a result, the horizontal leg force $F_{x}=\frac{m g}{y_{0}} x_{n o w}$ changes with the current position $x_{n o w}$ of the point mass, resulting in the equation of motion

$$
m \ddot{x}_{\text {now }}=\frac{m g}{y_{0}} x_{\text {now }} .
$$

Because the legs are massless, the swing leg (blue solid line in Fig. 1A) does not influence the dynamics of the point mass. To approximate realistic swing timings, however, we model the swing leg rotation about the hip as a damped oscillator that asymptotically converges to a target orientation (blue dashed line),

$$
J \ddot{\varphi}+r \dot{\varphi}+k \varphi=\varphi_{r e f},
$$

where $J=2.4 \mathrm{kgm}^{2}$ is the assumed swing leg inertia, $k=726 \mathrm{Nm} / \mathrm{rad}$ is the rotational stiffness, $b=0.5$ is the damping coefficient (values for $J, k$ and $b$ chosen to mimic human swing dynamics in walking [23] [24], with $\omega_{0}=\sqrt{k / J}$ and $\left.r=2 J \omega_{0} b\right), \varphi$ is the swing leg angle with respect to the vertical, and $\varphi_{\text {ref }}=\arctan \left(\frac{x_{T}}{y_{0}}\right)$ is its target value corresponding to a foot placement $x_{T}$. The target $x_{T}$ is set by the dynamic balance controller (section III-A). The swing leg touches down if $\left|\varphi-\varphi_{\text {ref }}\right|<1$ deg, subject to three conditions: the leg length does not exceed $l_{\max }=1 \mathrm{~m}$, the point mass has moved past the upright position, and the swing leg steps in front of the stance leg in the direction of movement.

Once in double support (Fig. 1B), both legs develop forces to support the point mass. We model the load sharing in the vertical force component as two linear functions in $x$ whose endpoints are defined by the leg length constraint (Fig. 2). This ensures symmetric dynamics with respect to the mid-stance $x_{m i d}=L_{\text {step }} / 2$ of double support, allowing the model to reverse its motion without creating dynamic inconsistencies. (Note that the force jumps are much smaller if compared to the force jumps implied by the traditional LIPM changing from one stance to the other.) The corresponding horizontal forces are $F_{x}^{h}=m g \frac{x_{\max }-x_{\text {now }}}{L_{\text {tot }}} \frac{x_{\text {now }}}{y_{0}}$ and $F_{x}^{f}=m g\left(1-\frac{x_{\text {max }}-x_{\text {now }}}{L_{\text {tot }}}\right) \frac{x_{\text {now }}-L_{\text {step }}}{y_{0}}$ for the hind and front leg, respectively, where $L_{t o t} \stackrel{y_{0}}{=} 2 x_{\max }-L_{\text {step }}$ is the

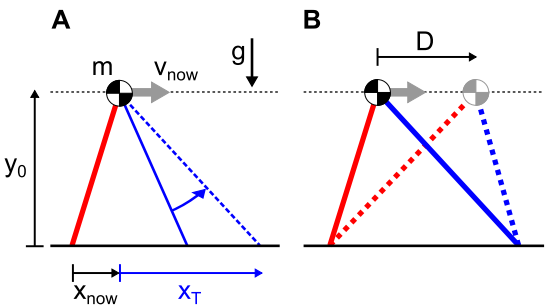

C

Fig. 1. Bipedal linear inverted pendulum model (bLIPM). (A) In single support, one leg is in stance (thick red) while the other (thin blue) swings to a commanded target $x_{T}$ (dotted blue). (B) Once the swing leg lands, the model stays in double support until the commanded length $D$ of the remaining double support goes to zero. (C) At that instant, the hind leg takes off and the model reenters single support. Additional parameters: $m=50 \mathrm{~kg}$ : mass, $g=9.81 \mathrm{~ms}^{-2}$ : gravitational acceleration, $y_{0}=0.8 \mathrm{~m}$ : COM height, $v_{\text {now }}$ : current speed.

feasible range of double support with $x_{\max }=\sqrt{l_{\max }^{2}-y_{0}^{2}}$ (Fig. 2). The resulting equation of motion simplifies to

$$
m \ddot{x}_{n o w}=\frac{m g}{y_{0}}\left(1-\frac{L_{\text {step }}}{L_{\text {tot }}}\right)\left(x_{\text {now }}-x_{\text {mid }}\right) .
$$

Similar to single support, the double support has the geometric midpoint $x_{m i d}$ as equilibrium position at which the net horizontal force vanishes. In contrast to single support, however, the model remains in double support for small deviations from this position if $L_{\text {step }}>L_{t o t}$, in which case the net horizontal force is a restoring one (Eq. 3). The equilibrium is not asymptotically stable as the model does not include damping and, following a deviation, it sways about $x_{m i d}$ in perpetuity. We avoid adding a damping controller to remedy this effect, and consider swaying in double support as standing in our model.

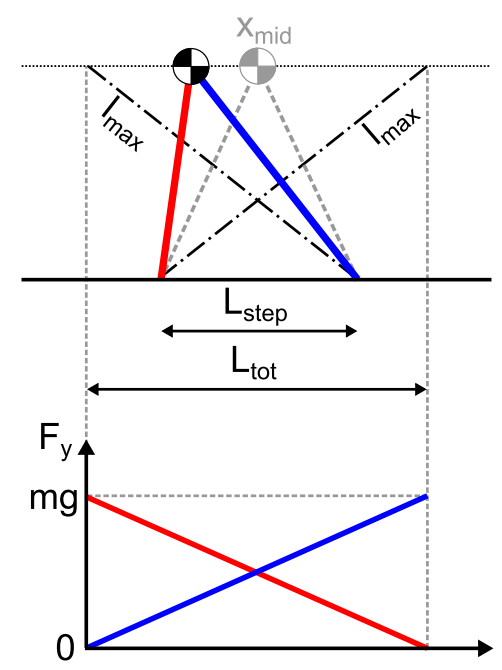

Fig. 2. Load sharing in double support. Over an interval that is defined by the maximum leg length $l_{\max }$ and can be larger than the step length $L_{s t e p}$, weight support $F_{y}=m g$ shifts linearly between the legs with equal support at mid-stance $x_{m i d}$.

The double support phase ends when the commanded length $D$ (section III-B) of the remaining double support goes to zero. Depending on the direction in which the point 
A

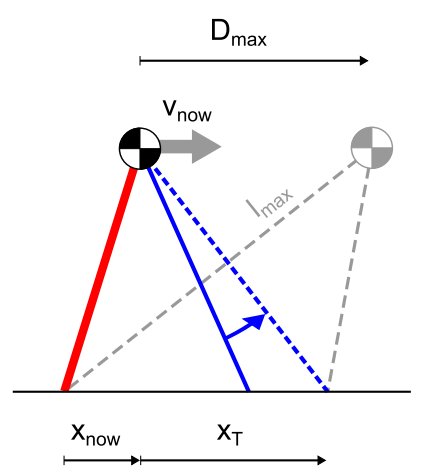

B

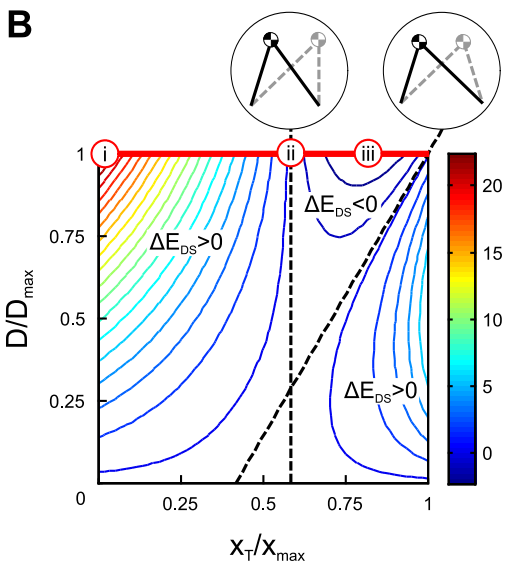

C

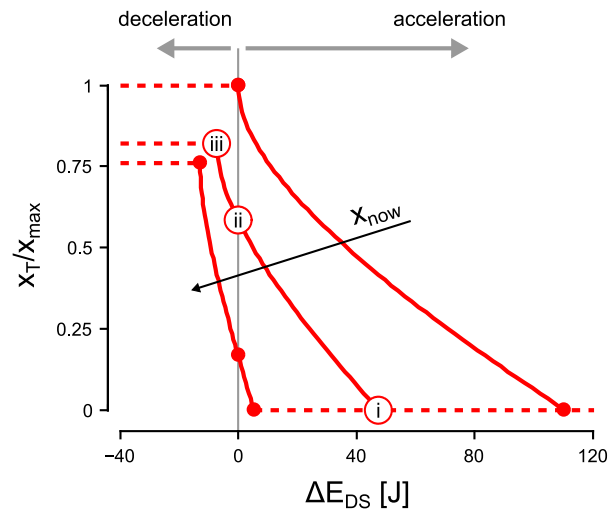

Fig. 3. Control strategy in single support. (A) Control inputs are the speed $v_{n o w}$ and position $x_{n o w}$ of the COM, and the desired speed $v^{*}$. The controller determines the swing leg target $x_{T}$, assuming that the touch down occurs immediately and that $v^{*}$ is to be reached by the end of double support. (B) The resulting change in energy $\Delta E_{D S}$ during double support is shown for an example value $x_{n o w}=0.25$ and all possible swing targets $x_{T}$ and double support lengths $D$. The black dotted lines mark zero energy change. The maximum (i) and the minimum (iii) of $\Delta E_{D S}$ occur at $D=D_{m a x}$, defining the control strategy (red line). (C) The control law adapts with progression $x_{n o w}$ of the COM.

mass is moving at that time, the respective hind leg switches to swing, the other leg takes over the whole body weight, and the model reenters single support (Fig. 1C).

Although the bLIPM is confined to horizontal motions, it can fail to balance the point mass during single support. If the stance leg extends to $l_{\max }$ before the swing leg reaches its target position, the model gets airborne. While for general biped locomotion this can mean either a transition to running or a fall, we assume the latter for the bLIPM as it does not model flight.

\section{DynAmic BALANCE CONTROLleR}

Similar to the original LIPM controller developed by [15], our dynamic balance controller uses three key inputs. The first two inputs are the horizontal position $x_{n o w}$ and speed $v_{\text {now }}$ of the COM. The third input is the target speed $v^{*}$. In contrast to previous approaches that set the target speed for the geometric midpoint in single support, we aim for this speed at the end of double support. We shift to double support because bLIPM can stand in this phase while tolerating disturbances. We focus on the end of double support because it maximizes the performance of the dynamic balance controller.

Although we aim for $v^{*}$ at the end of double support, we do not pre-plan trajectories. At any time, the dynamic balance controller seeks the immediate action required to accomplish the target speed goal without considering future interventions. This approach allows to react to unexpected perturbations; it requires however that the control strategy changes between single and double support.

\section{A. Single support strategy}

In single support, the dynamic balance controller tries to achieve the target speed $v^{*}$ by the end of double support assuming that the swing leg touches down immediately. Figure $3 \mathrm{~A}$ shows the biped model in single support with the COM position $x_{n o w}$ and speed $v_{\text {now }}$. The left leg (red) is in stance and the right leg (blue) is in swing, tracking the reference position $x_{T}$ (dashed) that is defined by the dynamic balance controller. To reach the speed $v^{*}$, a net change

$$
\Delta E_{T}=\frac{1}{2} m\left( \pm v^{* 2}-v_{n o w}^{2}\right)
$$

in energy is required between now and the end of double support $\left(+\right.$ or - if $v^{*}$ points in the direction of $v_{\text {now }}$ or the opposite one). This net energy change has to be realized entirely in double support if the swing leg touches down immediately.

The change $\Delta E_{D S}$ in energy that can be realized in double support for different combinations of target positions $x_{T}$ and double support lengths $D$ is

$$
\begin{aligned}
\Delta E_{D S} & \left(x_{n o w}, x_{T}, D\right) \\
= & \int_{x_{\text {now }}}^{x_{\text {now }}+D} F_{x} d x \\
= & \frac{m g}{y_{0}} \frac{D\left(x_{\text {max }}-x_{\text {now }}-x_{T}\right)\left(D+x_{n o w}-x_{T}\right)}{2 x_{\text {max }}-x_{\text {now }}-x_{T}} .
\end{aligned}
$$

Figure 3B shows an example contour plot of this function at a particular COM position $x_{\text {now }}=0.4 x_{\max }$ for the value ranges of $0 \leq x_{T} \leq x_{\max }$ and $0 \leq D \leq D_{\max }$ that respect the leg length constraint $l \leq l_{\max } . \Delta E_{D S}$ reaches both its maximum and its minimum (denoted by $\mathrm{i}$ and iii in Fig. 3B) for the maximum double support length $D_{\max }=x_{\max }-$ $x_{n o w}$. To maximize its performance, the dynamic balance controller assumes $D=D_{\max }$ in double support when providing target positions $x_{T}$ for the swing leg (red thick line in Fig. 3B). (Note that the minimum of $\Delta E_{D S}$ switches to small $D / D_{\max }$ for small $x_{n o w}$. But because dissipation can be re-adjusted in the subsequent double support while propulsion cannot, our controller provides placement targets based on the assumption $D=D_{\max }$.)

The target $x_{T}$ is calculated from $\Delta E_{D S}\left(x_{n o w}, x_{T}, D\right)=$ $\Delta E_{T}$, leading to a quadratic equation with only one solution, $x_{T}\left(\Delta E_{T}\right)=\left(-b_{1}-\sqrt{b_{1}^{2}-4 a_{1} c_{1}}\right) / 2 a_{1}$, in the permitted 
A

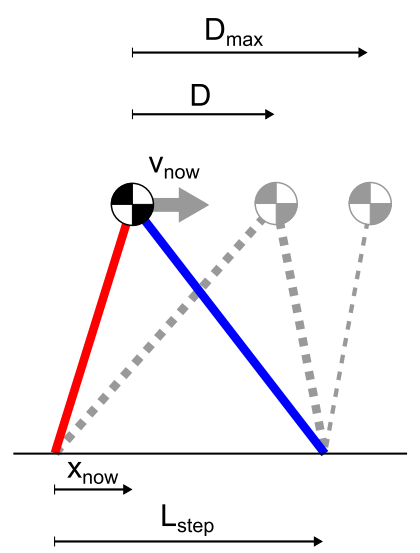

B

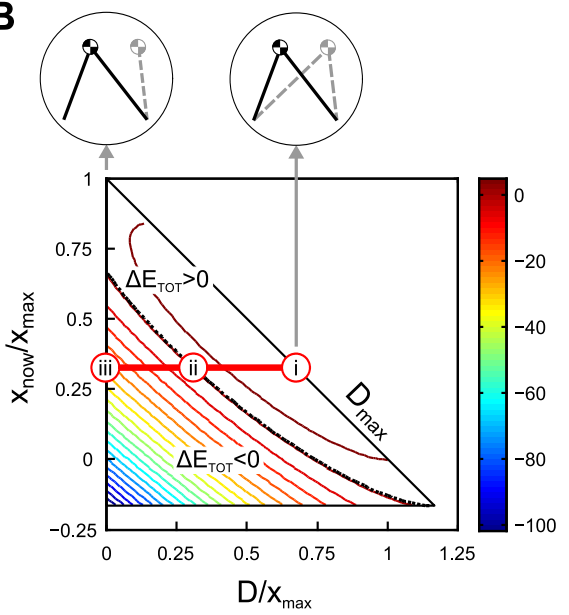

C

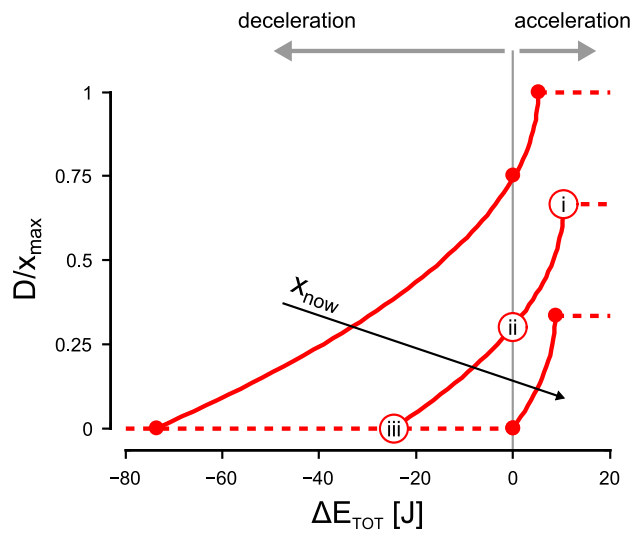

Fig. 4. Control strategy in double support. (A) Control inputs are the speed $v_{n o w}$ and position $x_{n o w}$ of the COM, the step length $L_{s t e p}$, and the target speed $v^{*}$. The controller determines the remaining length $D$ to be covered in double support, assuming that the remainder until $D_{\max }$ will be covered in single support. (B) The resulting change in energy $\Delta E_{T O T}$ is shown as a contour plot for all possible values of $D$ and $x_{n o w}$. The maximum (i) and minimum (iii) change in energy are always achieved for $D=D_{\max }$ and $D=0$, respectively.(C) Control laws adapt with progression $x_{n o w}$.

parameter range. The constants are $a_{1}=m g\left(x_{\text {max }}-x_{n o w}\right)$, $b_{1}=m g\left(x_{\max }-x_{\text {now }}\right)\left(-2 x_{\max }+x_{\text {now }}\right)+\Delta E_{T} y_{0}$, and $c_{1}=m g x_{\max }\left(x_{\max }-x_{\text {now }}\right)^{2}-\Delta E_{T} y_{0}\left(2 x_{\max }-x_{\text {now }}\right)$.

The solution forms a monotonic relationship between $\Delta E_{D S}$ and $x_{T}$. The dynamic balance controller uses this solution to provide target positions,

$$
x_{T}=\left\{\begin{array}{cl}
0, & \Delta E_{T}>\Delta E_{D S}^{\max } \\
x_{T}\left(\Delta E_{T}\right), & \Delta E_{D S}^{\min } \leq \Delta E_{T} \leq \Delta E_{D S}^{\max } \\
x_{T, E_{D S}^{\min }}, & \Delta E_{T}<\Delta E_{D S}^{\min }
\end{array}\right.
$$

where target values $x_{T}$ are clipped if the required energy change $\Delta E_{T}$ falls outside of the range of achievable changes $\Delta E_{D S}$. The control authority decreases with increasing $x_{n o w}$ (Fig. 3C), making immediate stepping a valuable strategy.

\section{B. Double Support Strategy}

Once in double support, the dynamic balance controller adapts the target distance $D$ of the remaining double support to accommodate required energy changes $\Delta E_{T}$. Figure $4 \mathrm{~A}$ shows the model in double support. Both legs contact the ground and the biped weight shifts between the two legs with the position $x_{\text {now }}$ (compare section II and Fig. 2). As in single support, the change in energy $\Delta E_{T}$ required to reach $v^{*}$ is given by Eq. 4 , and the dynamic balance controller tries to achieve this change at the maximum remaining distance $D_{\max }$ of double support. If $\Delta E_{D S}=\Delta E_{T}$ assuming maximum double-support length, no intervention occurs. However, if the two values differ, for instance when an unexpected push slows down the biped, the controller adapts the target distance $D$ of the remaining double support, assuming that the distance $D_{\max }-D$ will be covered in a subsequent single support. (Keeping $D_{\max }$ as reference point ensures consistent control policies in the alternating support phases.)

The total change in energy $\Delta E_{T O T}=\Delta E_{D S}+\Delta E_{S I S}$ until reaching $D_{\max }$ is given by

$$
\begin{aligned}
& \Delta E_{\text {TOT }}\left(x_{\text {now }}, L_{\text {step }}, D\right)= \\
& \quad \frac{m g}{y_{0}} \frac{D\left(x_{\text {max }}-L_{\text {step }}\right)\left(D-L_{\text {step }}+2 x_{\text {now }}\right)}{L_{\text {tot }}}+ \\
& \frac{m g}{2 y_{0}}\left[\left(x_{\text {max }}-L_{\text {step }}\right)^{2}-\left(D-L_{\text {step }}+x_{\text {now }}\right)^{2}\right]
\end{aligned}
$$

where $\Delta E_{D S}$ is the actual double-support contribution and $\Delta E_{S I S}$ is the remaining single-support contribution. Figure 4B shows an example contour plot of this function for a particular step length $L_{\text {step }}=0.8 x_{\max }$, an additional input to the dynamic balance controller in double support. At any position $x_{n o w}$ of the point mass, $\Delta E_{T O T}$ reaches its minimum for $D=0$, which corresponds to immediately shifting to single support and putting all the body weight onto the front leg to maximize horizontal braking. Similarly, $\Delta E_{T O T}$ always reaches its maximum for $D=D_{\max }$, allowing the back leg to maximize horizontal pushing. These results hold for any step length $L_{\text {step }}$ (Eq. 7). The actual remaining distance $D$ is computed from $\Delta E_{T O T}\left(x_{n o w}, L_{\text {step }}, D\right)=\Delta E_{T}$, which again leads to a quadratic equation with the solution $D\left(\Delta E_{T}\right)=\left(-b_{2}+\right.$ $\left.\sqrt{b_{2}^{2}-4 a_{2} c_{2}}\right) / 2 a_{2}$ in the permitted parameter range and constants $a_{2}=\frac{-m g L_{s t e p}}{2 y_{0}\left(2 x_{\text {max }}-L_{\text {step }}\right)}, b_{2}=\frac{m g L_{\text {step }}\left(x_{\max }-x_{\text {now }}\right)}{y_{0}\left(2 x_{\max }-L_{\text {step }}\right)}$, and $c_{2}=\frac{m g}{2 y_{0}}\left(x_{\max }-x_{\text {now }}\right)\left(x_{\max }+x_{\text {now }}-2 L_{\text {step }}\right)-\Delta E_{T}$. The resulting output of the dynamic balance controller is

$$
D=\left\{\begin{aligned}
0, & \Delta E_{T}<\Delta E_{T O T}^{\min } \\
D\left(\Delta E_{T}\right), & \Delta E_{T O T}^{\min } \geq \Delta E_{T} \geq \Delta E_{T O T}^{\max } \\
D_{E_{T O T}^{\max },} & \Delta E_{T}>\Delta E_{T O T}^{\max }
\end{aligned}\right.
$$

The model switches to single support when this output reaches zero, which happens if either the model progresses until $x_{\text {now }}=x_{\max }$ or an earlier event causes $D\left(\Delta E_{T}\right)=0$ (Fig. 4C). 


\section{Reversal of Motion}

The single and double support control strategies are invertible. The model's single support dynamics are symmetric with respect to $x_{\text {now }}=0$. The same applies to the double support since the hind leg changes with respect to the direction of motion, $v_{\text {now }}$. In effect, the dynamic balance controller equally performs in forward and backward motion and the same equations and solutions hold assuming that positions and distances are positive in the current direction of motion.

\section{Controller Performance}

The dynamic balance controller seamlessly integrates generating steady-state gait patterns with reacting to disturbances. These behaviors emerge from the choices of step length and double support length that the controller makes to maintain balance. The gait patterns result in definite average speeds. Figure 5A shows a representative example. The biped model initially walks at $v^{*}=1.25 \mathrm{~ms}^{-1}$ (blue solid line). At $1.5 \mathrm{~s}, v^{*}$ is set to zero (orange dashed line) and the controller brings the model within two steps to a stable double support (shaded background). The model remains standing until, at $4.5 \mathrm{~s}$, a strong $350 \mathrm{~ms}$ push of $-60 \%$ bw (body weight) forces the biped out of balance into a backward motion that would reach COM speeds in excess of $-2 \mathrm{~ms}^{-1}$ (green dashed line). The controller reacts and, after reaching a maximum backward speed of $-1.5 \mathrm{~ms}^{-1}$, the model recovers with three corrective steps standing in double support. At 9s, both the target speed is set to $1.5 \mathrm{~ms}^{-1}$ and a $150 \mathrm{~ms}$ forward push of $160 \%$ bw occurs that would cause a total speed of about $4 \mathrm{~ms}^{-1}$. The controller corrects this overshoot and ensures that the model converges to steady-state walking at the target speed. The controller thus integrates the generation of walking gaits at user-defined speeds with the reaction to unexpected external and internal disturbances to the COM motion (see also supplementary video).

Two factors limit the performance of the balance controller. The first one is the maximum speed with which the legs can move in swing (Eq. 2). Figure 5B shows for different maximum torques $\tau_{\max }$ of the swing leg motion the maximum push the model can tolerate without falling down. The result in general confirms the notion that the push tolerance increases with faster swing legs. However, it also suggests that push recovery can benefit from properly coupling the dynamics of swing and stance leg. The result shows that very slow swing legs $\left(\tau_{\max }<270 \mathrm{Nm}\right)$ have a better push recovery than moderately faster ones $(270 m<$ $\tau_{\max }<570 \mathrm{Nm}$ ). This trend reversal occurs, because faster swing legs protract to their target position $x_{T}$ before the COM reaches the upright position in single support $\left(x_{n o w}<\right.$ 0 ), suppressing the touch down. In effect, they overshoot and then retract to $x_{T}$. By this time the COM has reached an excessive forward lean for the moderately faster swing legs, which in subsequent single supports results in a continued net acceleration, and eventual fall. Thus, the proper coupling between the swing and stance leg dynamics as induced by a push can be as important for dynamic balance as swing leg speed.

The second factor that limits our controller performance is an asymmetry in the double-support control strategy. Although the dynamic balance controller compensates for constant push and pull forces while moving forward, it compensates clearly less pull than push (Fig. 5C). The reason for this asymmetry is an asymmetry in the available strategies to resist push or pull. Pushes can be maximally resisted by putting the swing leg far out in single support $\left(x_{T}=x_{\max }\right)$ and transition in the subsequent double support immediately back to single support of the front leg, where the leg maximally brakes until it reaches the upright position $x_{n o w}=0$. By contrast, the model cannot use the dual strategy of putting the swing leg to $x_{T}=0$ in single support and then immediately transitioning in the ensuing double support back to single support of the hind leg for maximum acceleration until $x_{n o w}=x_{\max }$, because the double support strategy does not consider going back into the single support phase of the hind leg.

\section{CONCLUSiON}

We have extended the linear inverted pendulum model into a bipedal system (Figs. 1 and 2) and derived for it a gait controller (Figs. 3 and 4) that dynamically balances standing, walking at user-defined speeds, and the transition between these behaviors, while countering intermittent and compensating for permanent disturbances as long as they are compatible with the swing-leg dynamics placing the feet (Fig. 5).

Our dynamic balance controller combines the advantages of two alternative stepping strategies that have previously been identified with the LIPM. Kajita and colleagues use the LIPM to plan footholds for required accelerations and decelerations [20], and a ZMP controller to execute these footholds on complex humanoids [25]. Although known disturbances can in part be countered while maintaining these footholds by injecting a desired momentum about the COM of the humanoids [26], unknown disturbances cannot be dealt with in this approach if they push the ZMP outside of the polygon of support. For that case, capture points have been identified with the LIPM as an alternative stepping strategy that absorbs the disturbance and stops the robot [3]. Our results show that such a distinction between stepping strategies is not required for a bipedal system. Our controller seamlessly combines stepping to accelerate and decelerate with stepping to react to disturbances, including external (ground irregularities, pushes) and internal ones (robot inertias and other parameter mismatches). This performance is realized because lift-off decisions in double support are integrated into the control, which contrasts to previous attempts extending the LIPM into a bipedal system [20] [22], yet reflects human strategies of balance control in locomotion. In addition, because double support dynamics are part of bLIPM, the controller automatically generates walking patterns with biologically plausible step lengths and step times. 


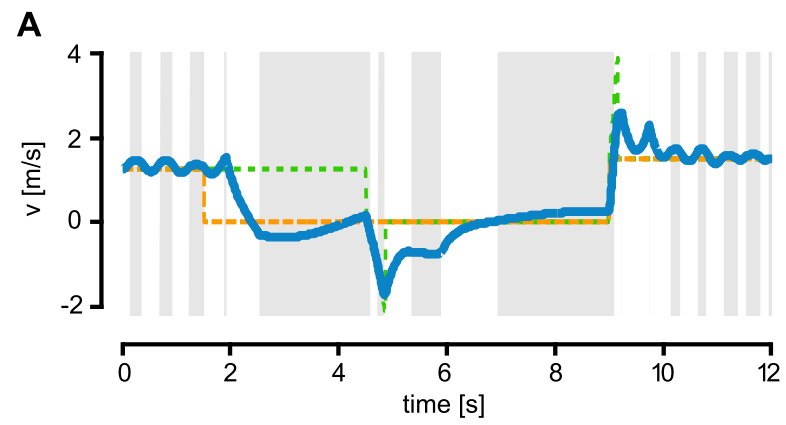

B

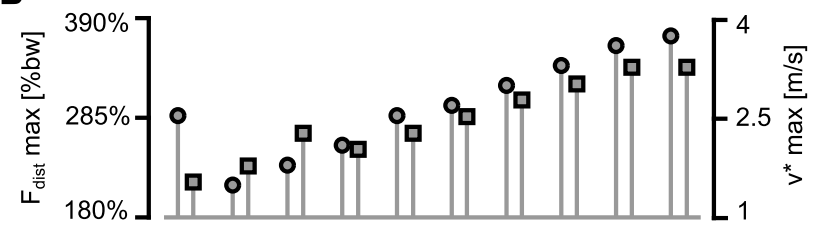

C

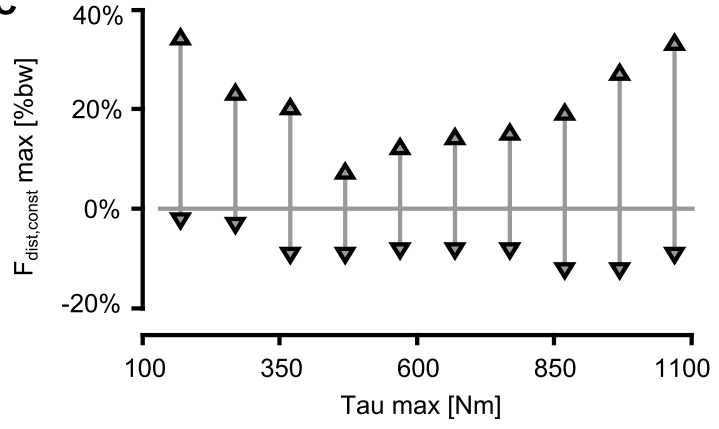

Fig. 5. Balance Controller Performance. (A) Example of intentional shifts between walking at target speeds and standing including push perturbations. Time traces show intended (dashed orange) and achieved COM speed (blue solid), and, for comparison, equivalent speed progression without controller intervention (green dashed). Shaded areas mark double supports. Pushes occur at $4.5 \mathrm{~s}(\mathrm{~F}=-60 \% \mathrm{bw}, \Delta t=350 \mathrm{~ms})$ and $9 \mathrm{~s}(\mathrm{~F}=160 \% \mathrm{bw}, \Delta t=150 \mathrm{~ms})$. (B) Relationships between maximum swing-leg torque and maximum push recovery in stance (circles, $v^{*}=0 \mathrm{~ms}^{-1}$, force applied for 100ms) and maximum steady state speed (squares) are shown. (C) Maximum constant push (positive) and pull forces (negative) that the controller can compensate while walking at $1.25 \mathrm{~ms}^{-1}$.

Despite its versatility, our balance controller also reveals limitations, which we seek to overcome in future work. We plan to generalize the double support strategy to include transitions back into single support of the hind leg without compromising the consistency of the control algorithm, and to extend the resulting controller to slope and 3D gait, exploring and exploiting the full theoretical potential of dynamic gait balance control based on the bLIPM.

\section{REFERENCES}

[1] D. Winter, "Human balance and posture control during standing and walking," Gait \& Posture, vol. 3, no. 4, pp. 193-214, December 1995.

[2] F. B. Horak and L. M. Nashner, "Central programming of postural movements: adaptation to altered support-surface configurations." $J$ Neurophysiol, vol. 55, no. 6, pp. 1369-1381, Jun 1986.

[3] J. Pratt, J. Carff, S. Drakunov, and A. Goswami, "Capture point: A step toward humanoid push recovery," in Proc. 6th IEEE-RAS Int Humanoid Robots Conf, 2006, pp. 200-207.

[4] B. Stephens, "Integral control of humanoid balance," in Proc. IEEE/RSJ Int. Conf. Intelligent Robots and Systems IROS 2007, 2007, pp. 4020-4027.
[5] D. A. Winter, A. E. Patla, F. Prince, M. Ishac, and K. Gielo-Perczak, "Stiffness control of balance in quiet standing." J Neurophysiol, vol. 80, no. 3, pp. 1211-1221, Sep 1998.

[6] B. Stephens, "Humanoid push recovery," in Proc. 7th IEEE-RAS Int Humanoid Robots Conf, 2007, pp. 589-595.

[7] A. V. Alexandrov, A. A. Frolov, and J. Massion, "Biomechanical analysis of movement strategies in human forward trunk bending. i. modeling," Biological Cybernetics, vol. 84, no. 6, pp. 425-434, 2001.

[8] M. Abdallah and A. Goswami, "A biomechanically motivated twophase strategy for biped upright balance control," in Proc. IEEE Int. Conf. Robotics and Automation ICRA 2005, 2005, pp. 1996-2001.

[9] A. G. Hofmann, "Robust execution of bipedal walking tasks from biomechanical principles," Ph.D. dissertation, Massachusetts Institute of Technology, Cambridge, MA, USA, 2006.

[10] J. E. Pratt and R. Tedrake, "Velocity-based stability margins for fast bipedal walking," in Fast Motions in Biomechanics and Robotics, 2006, vol. 340/2006, pp. 299-324.

[11] M. A. Townsend, "Biped gait stabilization via foot placement." $J$ Biomech, vol. 18, no. 1, pp. 21-38, 1985.

[12] C. E. Bauby and A. D. Kuo, "Active control of lateral balance in human walking." J Biomech, vol. 33, no. 11, pp. 1433-1440, Nov 2000.

[13] A. L. Hof, M. G. J. Gazendam, and W. E. Sinke, "The condition for dynamic stability," Journal of Biomechanics, vol. 38 , no. 1, pp. $1-8$, 2005.

[14] M. S. Redfern and T. Schumann, "A model of foot placement during gait," Journal of Biomechanics, vol. 27, no. 11, pp. 1339 - 1346, 1994.

[15] S. Kajita, K. Tani, and A. Kobayashi, "Dynamic walk control of a biped robot along the potential energy conserving orbit," in Proc. IEEE Int. Workshop Intelligent Robots and Systems '90. 'Towards a New Frontier of Applications' IROS '90, 1990, pp. 789-794.

[16] J. Rebula, F. Canas, J. Pratt, and A. Goswami, "Learning capture points for humanoid push recovery," in Proc. 7th IEEE-RAS Int Humanoid Robots Conf, 2007, pp. 65-72.

[17] D. L. Wight, E. G. Kubica, and D. W. L. Wang, "Introduction of the foot placement estimator: A dynamic measure of balance for bipedal robotics," Journal of Computational and Nonlinear Dynamics, vol. 3, no. 1, p. 011009, 2008.

[18] K. Yin, K. Loken, and M. van de Panne, "Simbicon: simple biped locomotion control," in SIGGRAPH '07: ACM SIGGRAPH 2007 papers. New York, NY, USA: ACM, 2007, p. 105.

[19] J. E. Pratt, B. Krupp, V. Ragusila, J. Rebula, T. Koolen, N. van Nieuwenhuizen, C. Shake, T. Craig, J. Taylor, G. Watkins, P. Neuhaus, M. Johnson, S. Shooter, K. Buffinton, F. Canas, J. Carff, and W. Howell, "The yobotics-ihmc lower body humanoid robot," in Proc. IEEE/RSJ Int. Conf. Intelligent Robots and Systems IROS 2009, 2009, pp. 410-411.

[20] S. Kajita, F. Kanehiro, K. Kaneko, K. Fujiwara, K. Yokoi, and H. Hirukawa, "A realtime pattern generator for biped walking," in Proc. IEEE Int. Conf. Robotics and Automation ICRA '02, vol. 1, 2002, pp. 31-37.

[21] J. E. Pratt and S. V. Drakunov, "Derivation and application of a conserved orbital energy for the inverted pendulum bipedal walking model," in Proc. IEEE Int Robotics and Automation Conf, 2007, pp. 4653-4660.

[22] B. Stephens and C. Atkeson, "Modeling and control of periodic humanoid balance using the linear biped model," in Proc. 9th IEEERAS Int. Conf. Humanoid Robots Humanoids 2009, 2009, pp. 379384.

[23] J. Perry, Gait Analysis : Normal and Pathological Function. Delmar Learning, January 1992.

[24] D. A. Winter, Biomechanics and Motor Control of Human Movement. Wiley, August 2004.

[25] S. Kajita, F. Kanehiro, K. Kaneko, K. Fujiwara, K. Harada, K. Yokoi, and H. Hirukawa, "Biped walking pattern generation by using preview control of zero-moment point," in Proc. IEEE Int. Conf. Robotics and Automation ICRA '03, vol. 2, 2003, pp. 1620-1626.

[26] _ _ "Resolved momentum control: humanoid motion planning based on the linear and angular momentum," in Proc. IEEE/RSJ Int. Conf. Intelligent Robots and Systems IROS 2003, vol. 2, oct. 2003, pp. 1644 -1650 . 